\section{Length of Stay in Long-Term Care Settings}

Charlene H. Chu

Lawrence S. Bloomberg Faculty of Nursing, University of Toronto, Toronto, ON, Canada KITE-Toronto Rehabilitation Institute, University Health Network, Toronto, Canada

\section{Synonyms}

Duration of stay; Length of stay since admission; Stay length; The period of stay in a long-term care facility; Time elapsed since admission

\section{Definition}

Length of stay refers to the time between an individual's admission into the long-term care setting until death or discharge from the facility.

\section{Overview}

Length of stay (LoS) has been extensively researched across the healthcare continuum, including long-term care homes, as a meaningful outcome measure and a potential target for quality improvement activities and programs. Length of stay can be measured in days, months, years, or any other unit of time and measured from a specific point after admission until either death, transfer to another setting, or discharge (Moore et al. 2019). LoS has implications on care planning and budgetary decisions in long-term care and across the health care continuum. As a result, there is a large body of evidence related to examining the factors that affect $\operatorname{LoS}$ in addition to investigating models that predict an individual's expected LoS. Overall, LoS is typically found in administrative data sets and is used as an indicator for healthcare system function; for example, shorter LoS across the sector rapidly increases admission rates into long-term care, whereas a longer LoS would reduce the number of placements available for new admissions resulting in longer wait times and increased demands for a placement and community services. Longer LoS may also indicate a need for innovative strategies to meet care challenges, for example, the creation of new care models such as different staff skill complements within long-term care homes (e.g., regulated staff and unregulated staff ratios) or shifting services for older adults to include assisted living, community-based alternative programs, and public and private partnerships (Sloane et al. 2014). LoS can also be used to compare long-term care homes and identify variation of LoS based on resident profiles. Many variables influence $\operatorname{LoS}$ as a measure of longterm care home quality (e.g., individual characteristics, rigor of research methodology, study sample, etc.). Accordingly, there are differing stay lengths around the world to reflect the different 
health care systems, policies, models of care, time periods, and aging populations. As such, LoS estimates will constantly fluctuate to reflect changes in these important contextual variables.

\section{Key Research Findings}

Many research studies and reports support that older adults admitted into long-term care are more frail, medically complex, and dependent than previous cohorts (Boyd et al. 2011; Moore et al. 2012; Ontario Long-term Care Association [OLTCA], 2018; Statistics, 2017). There is a general sense that LoS in long-term care is shorter given the increased impairment of admitted older adults (Hoben et al. 2019); however, the international evidence shows a large variation in LoS between residents in the same country and between countries (Honinx et al. 2019; Moore et al. 2019). There is also an increasing proportions of younger individuals with multiple chronic conditions and disabilities entering long-term care facilities which will impact overall LoS (OLTCA 2019).

LoS estimates from various countries are highly heterogeneous between low-, middle-, and high-income countries. For example, the mean LoS in a nursing home in the USA was reported to be 2.29 years (Bercovitz et al. 2009) compared to 5.5 years in Brazil (Viana et al. 2015). A large variation in current trends worth noting includes national longitudinal studies that show increasing care needs in long-term care in Canada (Hoben et al. 2019) which decreases LoS, while LoS remains constant in Australia (Andrews-Hall et al. 2007), increasing in Brazil (Viana et al. 2015), and fluctuating in Scotland (Information Services Division 2016). A closer examination reveals difference in the resident populations: In Brazil, 58.5\% of residents were living in long-term care facilities for 5 years or more (Viana et al. 2015), which is much higher than other countries. While in the USA, the National Nursing Home Survey reported that $88 \%$ of these residents stayed less than 5 years (Bercovitz et al. 2009). In six European countries, a large variation in LoS was also present and was associated to factors prior to admission including resources available to keep older adults at home (Moore et al. 2020b). Differences can also be seen within countries to reflect policies, access to care, and epidemiological trends of specific geographical areas. For example, three different provinces in Canada reported stay lengths that ranged between 1.07 to 2.4 years (Hoben et al. 2019).

\section{Factors Associated with LoS in Long- term Care}

There is a considerable amount of research examining the factors that influence or are associated with LoS. A recent systematic review found that shorter LoS were associated with characteristics related to end of life. Results identified three resident characteristics had strong evidence to support their association with shorter LoS: shortness of breath, receipt of oxygen therapy, and admission into a nursing home compared to a residential care home (Moore et al. 2019). The review also found nine resident characteristics had moderate evidence to support their association with shorter LoS: diagnosis of cancer, increased contact with primary care, poor general health, poor mobility, low body mass index or malnutrition, poor physical functioning, presence of pressure ulcers, older age, and being male (Moore et al. 2019). Another study found that having a chronic illness was associated with statistically significant differences with the LoS being the shortest for patients with diagnoses of cancer or lung disease (Kelly et al. 2010). In Canada, a large retrospective cohort study found three variables were related to shorter LoS: higher physical dependency, higher level of cognitive impairment, or health instability were associated with shorter LoS in parts of Canada (Hoben et al. 2019). Another study using a national US survey reported relationships between demographic variables and LoS including that men have shorter LoS compared to women; older adults who were married at the time of placement had a shorter median LoS compared to those who were unmarried, and the highest quartile of net worth $(>\$ 150,000)$ had a shorter LoS than those in lowest quartile (Kelly et 
al. 2010). A study investigated the resident, facility, and country characteristics associated with LoS in LTC facilities across six European countries using a large, representative sample of residents (Moore et al. 2020b). The authors used proportional hazards models and found older age at admission, being married/in a civil partnership at time of death, having cancer at time of death, and admission from a hospital or another LTC facility were associated with shorter LoS across all countries (Moore et al. 2020b).

At a macro level, it is relevant to note that cross-country international comparisons of LoS are challenging due to the use of different terminologies referring to "long-term care" and by extension, the variation of individuals who enter these long-term care facilities. Terms such as assisted living, nursing home, care home, residential care home, skilled nursing facility, or hospice are used interchangeably despite jurisdictional variations in what services are offered and how LoS may be reported and calculated between sectors. For instance, Brazil's LoS (5 years) is significantly longer than other countries due to a significant percentage of functionally independent older individuals as Brazilian long-term care homes appear to more closely resemble assisted living facilities (Viana et al. 2015).

Additionally, the sociopolitical context that includes policies and services that support agingin-place community-based supports will impact LoS. Differences between and within countries are influenced aging-in-place supports and increasing equity with increased access to primary care (Viana et al. 2015). Access to community care services that keeps older adults at home for a longer period will shorten their LoS in long-term care because they will enter the facility later in their illness trajectory (Doupe et al. 2016). The extent to which populations have access to community or home care services can influence when and who enters long-term care facilities.

A study in Canada found that policies directed at aging in the community reduced the overall LoS in long-term care homes, but that such residents are entering long-term care homes with more complex care needs (Hoben et al. 2019). Globally, there is a vested interest by governments to manage the increasing demand for long-term care and the needs of older adults. One strategy is to reduce the reliance on long-term care homes and enable older people to live at home for longer with the appropriate supports in order to reduce lifetime use of long-term care. Given this background, it is essential to understand that the LoS estimate is determined by the broader healthcare system, culture, other variables that supply contextual variables. Rather, a comprehensive examination that includes contextual information about the broader healthcare system is essential to make appropriate comparisons between countries.

\section{The Association Between LoS and Other Outcomes}

A systematic review of polypharmacy of residents in long-term care reported an inverse association with LoS in LTCH (Jokanovic et al. 2015). A study across six European countries examining the relationship between $\operatorname{LoS}$ and end-of-life care found that longer LoS in long-term care homes were associated with increased quality of care and comfort in the last month and week, respectively, prior to death (Moore et al. 2020a). Longer LoS was also related to the increased probability of having advance directives and a power of attorney in place (Moore et al. 2020a). In another study, a longer LoS in a long-term care facility as well as having dementia and being on hospice care were most strongly associated with poor oral hygiene assessments and hygiene scores (Zimmerman et al. 2017). Longer LoS remained an independent risk factor for poor oral hygiene in older adults living in long-term care to suggest that ongoing poor care reduced overall on oral hygiene (Zimmerman et al. 2017). Lastly, investigators found that the chance of receiving physiotherapy slightly diminished as more time elapsed after admission $(n=600)$ (Leemrijse et al. 2007).

\section{Relevance and Implications}

LoS in long-term care facilities is important to many stakeholders because $\operatorname{LoS}$ is related to 
transfer, early death, and long-term survival. Determining LoS, as well as the risk factors associated with $\mathrm{LoS}$, is important to the healthcare team, residents, and their families. From the perspectives of long-term care home administrators, decision makers' perspectives (e.g., government departments), and industries (e.g., insurance, technology development, real estate), the overall pattern of LoS in long-term care affects important budgetary decisions, care policies, and priorities, as well as planning the allocation of resources.

The duration of time residents spend in a longterm care facility before death has important clinical and policy implications. Case in point, if the LoS is longer than the expected norm in the geographical region, then organizational and policy directives may prioritize resident re-enablement programs and focus resources to improve mobility and functional status. Whereas if LoS is generally brief and closer to the end of life, then access to quality palliative and end-of-life care should be highlighted (Kelly et al. 2010; Moore et al. 2019). There are also other organizational consequences related to LoS. For example, in one UK study (Xie et al. 2005) found that $64 \%$ of admissions into long-term care facilities will stay an average of 2.1 years ( 784 days), with $25 \%$ of older adults staying longer than 2.1 years and 10\% living longer than 4.1 years. As time passes, there will be a reduction in the places that are available for new admissions since many beds will be occupied which will have serious long-term financial and care implications (Xie et al. 2005).

\section{Future Directions of Research}

Future research must consider the broader context including long-term care policies and funding, changing resident outcomes and health profiles, staffing outcomes, and quality of care metrics. Studies are required to continually update the $\mathrm{LoS}$ in long-term care due to changing care models, epidemiological trends, and advances in medicine and technology. This section will highlight some areas for future research directions:
1. The impact of new models of care on LoS: New and innovative models of care are being implemented in various countries around the world (e.g., village models, Butterfly homes, The Eden Alternative, long stay beds, convalescent care) to reflect changing needs, philosophies of care, increased access to care, and to enhance collaboration and improve care for their local populations. The short- and longterm effects of these new models of care on resident-level and organizational-level outcomes, including LoS, will be important to elucidate.

2. Examination of LoS within the context of new technologies: Advances in technology innovations and the adoption of these technologies into the community to support ageing-inplace (e.g., smart homes) as well as monitoring technologies inside long-term care facilities can impact LoS (e.g., remote monitoring systems). Technology-enabled long-term care services such as health information technology, telemedicine, assistive technology, online resources, and apps can support independent living (Chambers et al. 2016; see chapters "Telemedicine"; "Technology and Telemedicine"; "Gerontechnology"; - "Telehealth"; "Telenursing"; > "Telerehabilitation"). Wearable sensors and environmental sensors can remotely monitor individuals and collect longitudinal real-time data that can supplement and inform clinician assessments (see chapters $>$ "Managing LongTerm Conditions: Wearable Sensors and IoTBased Monitoring Applications"; "Wearable Technology"). Development of technologyenabled long-term care services is especially important in some public health crises. For example, the significant proportion of casualties from long-term care facilities during the COVID-19 pandemic has resulted in this sector being deemed "ground zero" of the global crisis (Barnett and Grabowski 2020; see chapter " "COVID-19 and Healthy Aging").

This is an emerging area of research and more evidence is needed about the design and implementation of these technologies into long-term care facilities, how they influence 
care practices and resident level outcomes, including length of stay, is warranted.

3. The impact of LoS trends on long-term care staff and their ability to provide care: The increasing care needs of older adults in longterm care with shorter LoS may have major implications for care staff. Research is required to understand these changes to staff's workload, the quality of care they can provide, and quality of their work life (e.g., burnout, job satisfaction, job stress, turnover). Some evidence indicates that shorter LoS negatively impacted staff's connection and knowledge of the needs, preferences, and essence of the dying resident (McCleary et al. 2018). Shorter LoS may also shift how staff prioritize their work (e.g., less emphasis on physical activity, mobility, rehabilitation) and may approach their care in a more supportive role. Exploring how LoS trends may impact quality of care, like end of life care for residents with dementia, the extent to which person-centered care can be delivered, and staff outcomes like job satisfaction or intensity of work, is important to support the staff in these settings.

4. Cross-cultural comparisons of LoS: There is a need for a measurement infrastructure which accounts for performance metrics and consensus definitions to facilitate international robust cross-cultural comparisons of LoS (Papanicolas et al. 2013). A framework or infrastructure will allow the aggregation of LoS data despite geographical differences including different cultural norms, payment schemes, and access to long-term care facilities. Such an infrastructure should be applicable and ecologically viable to low income and middle-income countries (i.e., feasible to countries without electronic health records). An infrastructure of common data elements that can facilitate accurate cross-cultural comparisons can offer a unique tool for international resident-level and organizational-level comparisons and for policymakers interested in understanding whether their health system is performing as well as it could, and in identifying the scope for improvement. WE-THRIVE (Corazzini et al. 2019) is an international consortium of nurse researchers who are focused on developing common data elements for this purpose, but work in this area is still in its infancy.

\section{Summary}

LoS is an indicator of the healthcare system and has been used as a variable, outcome, and quality metric in various settings across the healthcare continuum, including long-term care. LoS can be influenced by many variables including epidemiological trends, care models, and specific clinical settings with different populations and mandates (e.g., residential care, nursing homes, etc.), health care systems and policies, access to care, and advances in medicine and technology around the globe. As such, a considerable amount of robust research has been conducted to identify the variables that are associated with LoS. This being said, advances in modern medicine are also extending the years of life and increasing prevalence of co-morbidities and disabilities which will change the profile of individuals entering longterm care. More research is required to better understand the impact of care models and technological innovations on LoS, as well as the effect a changing LoS has on staff's ability to care. Lastly, there is a need for a measurement infrastructure that can enable cross-cultural comparisons of stay lengths in long-term care facilities and inform policy makers and financial and resource allocation.

\section{Cross-References}

\author{
- Advance Care Planning \\ - Alternative Models of Long-term Care \\ - Care Management \\ COVID-19 and Healthy Aging \\ - Health-Care Financing \\ - Health-Care Financing \\ National Long Term Care Survey \\ Quality of Care \\ Technology-Enabled Long-Term Care Services \\ and Supports (T-eLTCSS) in Home Settings
}


Telehealth

$\checkmark$ Telemedicine

\section{References}

Andrews-Hall S, Howe A (2007) The dynamics of residential aged care in Australia: 8-year trends in admission, separations and dependency. Aust Heal Rev 31:611

Barnett ML, Grabowski DC (2020) Nursing homes are ground zero for COVID-19 pandemic. In JAMA Health Forum 1(3):e200369-e200369. American Medical Association. https://jamanetwork.com/channels/ health-forum/fullarticle/2763666?utm_campaign= articlePDF\%26utm medium=articlePDFlink\%26utm source $=$ articlePDF\%26utm_content $=$ jama.2020.10419

Bercovitz A, Dwyer L, Jones A, Strahan G (2009) The National Nursing Home Survey; 2004 overview. Vital Health Stat 167:1-155

Boyd M, Broad JB, Kerse N, Foster S, von Randow M, Lay-Yee R, Chelimo C, Whitehead N, Connolly MJ (2011) Twenty-year trends in dependency in residential aged care in Auckland, New Zealand: A descriptive study. J Am Med Dir Assoc 12:535-540. https://doi. org/10.1016/J.JAMDA.2011.01.014

Chambers R, Schmid M, Birch-Jones J (2016) Digital healthcare: The essential guide. Otmoor Publishing, Oxford

Corazzini KN, Anderson RA, Bowers BJ, Chu CH, Edvardsson D, Fagertun A, ... Siegel EO (2019) Toward common data elements for international research in long-term care homes: Advancing personcentered care. Journal of the American Medical Directors Association 20(5):598-603

Division Information Services Division of National Services Scotland (2016) A National Statistics Publication for Scotland Care Home Census for Adults in Scotland 2006-2016, Edinburgh

Doupe M, Finlayson G, Khan S, Yogendran M, Schultz J, McDougall C, Kulbalba C (2016) Supportive Housing for Seniors: Reform Implications for Manitoba's Older Adult Continuum of Care Winnipeg, MB. Manitoba Centre for Health Policy, Spring. http://mchp-appserv. cpe.umanitoba.ca/reference/Supportive \%20Housing_ report_web.pdf

Hoben M, Chamberlain SA, Gruneir A, Knopp-Sihota JA, Sutherland JM, Poss JW, Doupe MB, Bergstrom V, Norton PG, Schalm C, McCarthy K, Kashuba K, Ackah F, Estabrooks CA (2019) Nursing home length of stay in 3 Canadian Health Regions: temporal trends, jurisdictional differences, and associated factors. J Am Med Dir Assoc. https://doi.org/10.1016/J. JAMDA.2019.01.144

Honinx E, Van Dop N, Smets T, Deliens L, Van Den Noortgate N, Froggatt K, Gambassi G, Kylänen M, Onwuteaka-Philipsen B, Szczerbińska K, Van Den Block L (2019) Dying in long-term care facilities in Europe: the PACE epidemiological study of deceased residents in six countries. BMC Public Health 19:1199. https://doi.org/10.1186/s12889-019-7532-4

Jokanovic N, Tan ECKC, Dooley MJ, Kirkpatrick CM, Bell JS, Jokanovic BPharm N, Tan ECKC, Dooley MJ, Kirkpatrick CM, Simon Bell J (2015) Prevalence and factors associated with polypharmacy in long-term care facilities: a systematic review. JAMDA 16:535.e1-535.e12. https://doi.org/10.1016/ j.jamda.2015.03.003

Kelly A, Conell-Price J, Covinsky K, Cenzer IS, Chang A, Boscardin WJ, Smith AK (2010) Length of stay for older adults residing in nursing homes at the end of life. J Am Geriatr Soc 58:1701-1706. https://doi.org/ 10.1111/j.1532-5415.2010.03005.x

Leemrijse CJ, de Boer ME, van den Ende CHM, Ribbe MW, Dekker J (2007) Factors associated with physiotherapy provision in a population of elderly nursing home residents; a cross sectional study. BMC Geriatr 7:7. https://doi.org/10.1186/1471-2318-7-7

McCleary L, Thompson GN, Venturato L, WicksonGriffiths A, Hunter P, Sussman T, Kaasalainen S (2018) Meaningful connections in dementia end of life care in long term care homes. BMC Psychiatry 18:307. https://doi.org/10.1186/S12888-018-1882-9

Moore DC, Keegan TJ, Dunleavy L, Froggatt K (2019) Factors associated with length of stay in care homes: a systematic review of international literature. BMC Syst Rev 8. https://doi.org/10.1186/s13643-019-0973-0

Moore DC, Payne S, Keegan T, Deliens L, Smets T, Gambassi G, Kylänen M, Kijowska V, OnwuteakaPhilipsen B, Van den Block L (2020a) Associations between length of stay in long term care facilities and end of life care. Analysis of the PACE cross-sectional study. Int J Environ Res Public Health 17:2742. https:// doi.org/10.3390/ijerph17082742

Moore DC, Payne S, Keegan T, Van den Block L, Deliens L, Gambassi G, Heikkila R, Kijowska V, Roeline Pasman H, Pivodic L, Froggatt K (2020b) Length of stay in long-term care facilities: a comparison of residents in six European countries. Results of the PACE cross-sectional study. BMJ Open.. https://doi.org/ 10.1136/bmjopen-2019-033881

Moore KL, Boscardin WJ, Steinman MA, Schwartz JB (2012) Age and sex variation in prevalence of chronic medical conditions in older residents of U.S. Nursing Homes. J Am Geriatr Soc 60:756-764. https://doi.org/ 10.1111/j.1532-5415.2012.03909.x

OLTCA (2018) This is long-term care 2018. Toronto, Ontario Long Term Care Association

Ontario Long Term Care Association (2019) This is longterm care. Toronto, Ontario Long Term Care Association

Papanicolas I, Kringos D, Klazinga N, Policy PS-H (2013) Health system performance comparison: new directions in research and policy. Health Policy (New York) 112:1-3

Sloane PD, Zimmerman S, D'Souza MF (2014) What will long-term care be like in 2040? N C Med J 75:326-330 
Statistics N (2017) National Center for Health Statistics (US). Health, United States, 2016: With Chartbook on Long-term Trends in Health. Hyattsville (MD): National Center for Health Statistics (US).

Viana B d M, Bicalho MAC, Moraes EN, Romano-Silva MA (2015) Twenty-four-year demographic trends of a Brazilian long-term care institution for the aged. J Am Med Dir Assoc 16:174.e1-174.e6. https://doi.org/ 10.1016/j.jamda.2014.11.012
Xie H, Chaussalet TJ, Millard PH (2005) A continuous time Markov model for the length of stay of elderly people in institutional long-term care. J R Stat Soc Ser A 168:51-61. https://doi.org/10.1111/j.1467985X.2004.00335.X

Zimmerman S, Austin S, Cohen L, Reed D, Poole P, Ward K, Sloane PD (2017) Readily identifiable risk factors of nursing home residents' oral hygiene: dementia, hospice, and length of stay. J Am Geriatr Soc 65:25162521. https://doi.org/10.1111/jgs.15061 